\title{
Diagnóstico del potencial de emprendimientos ecoamigables en la provincia de Tayacaja, Huancavelica, Perú
}

\author{
Diagnosis of the potential of eco-friendly \\ ventures in Tayacaja Province, Huancavelica, \\ Peru
}

\author{
(iD) 'Ronald Ortecho Llanos (iD) 'Dario Emiliano Medina Castro (iD) 'Damián Manayay Sánchez Gino \\ (iD) 'Gino Paúl Prieto Rosales (iD) ${ }^{2}$ Amyrsa Salgado Rodríguez
}

Universidad Nacional Autónoma de Tayacaja "Daniel Hernández Morillo". Pampas, Perú. Universidad de Camaguey Ignacio Agramonte Loynaz. Camaguey, Cuba.²

\section{Resumen}

El emprendedurismo tiene un papel preponderante en el desarrollo de las naciones, América Latina y el Perú, no son ajenas a estas oportunidades, su impulso implica negocios innovadores y arriesgados, por cuanto nacen de las oportunidades del entorno socioeconómico que en todo momento busca ser amigable con la sostenibilidad del ecosistema, para lo cual se requiere tener identificadas las potencialidades que este entorno ofrece, y como respuesta a esta necesidad, este diagnóstico se propuso como objetivo; identificar las posibilidades de emprendimientos eco amigable de la provincia de Tayacaja, previa valoración cualitativa y cuantitativa de las potencialidades sociales y eco sostenibles existentes. Entre los métodos y técnicas se emplearon: revisión documental, observación, entrevistas y encuestas en campo, fueron las técnicas de mayor significación para este estudio. Entre los principales resultados se destacaron que la biodiversidad de los ecosistemas, las identidades culturales y las actividades económicas representan aproximadamente el $60 \%$ de las potencialidades del emprendimiento eco amigable, la Asociación de productores y comerciantes, así como la Universidad, se convierten en potenciales soportes de la vigencia sostenible de estos emprendimientos.

Palabras clave: Emprendedurismo, emprendimiento, emprendimiento ecoamigable, potencialidades para el emprendimiento, diagnóstico para el emprendimiento.

Recibido:

Abril 2020

Aceptado: Junio 2020

\section{Abstract}

Entrepreneurship has a preponderant role in the development of nations, Latin America and Peru, they are not oblivious to these opportunities, their drive implies innovative and risky businesses, since they are born from the opportunities of the socioeconomic environment that at all times seeks to be friendly with the sustainability of the ecosystem, for which it is necessary to have identified the potentialities that this environment offers, and in response to this need, this diag- 
nosis was proposed as an objective; identify the possibilities of eco friendly ventures in the province of Tayacaja, after qualitative and quantitative assessment of the existing social and eco-sustainable potential. Among the methods and techniques used: documentary review, observation, interviews and field surveys, were the most significant techniques for this study. Among the main results, it was highlighted that the biodiversity of ecosystems, cultural identities and economic activities represent approximately $60 \%$ of the potential of eco-friendly entrepreneurship, the Association of producers and traders, as well as the University, become potential supports for the sustainable validity of these ventures. Keywords: Entrepreneurship, entrepreneurship, eco-friendly entrepreneurship, potential for entrepreneurship, diagnosis for entrepreneurship.

\section{INTRODUCCIÓN}

En un contexto económico caracterizado por una demanda hacia un consumo racional de los recursos y la producción de alimentos de manera sostenible, donde prima la demanda a la protección a los ecosistemas, resulta perentorio potenciar nuevos negocios, o transformar los ya existentes, teniendo como premisa la aplicación de la innovación y las tecnologías para emprender negocios amigables con el medio ambiente que a su vez constituyan palancas de desarrollo económico-social de las localidades y mejora de la calidad de vida de sus pobladores.

De acuerdo a lo expresado por (Schumpeter,1942) el emprendimiento significa, entre otros: reformar o revolucionar el patrón de producción al explotar una invención, o una posibilidad técnica no probada, para producir un nuevo producto o uno viejo de una nueva manera; proveer de una nueva fuente de insumos o un material nuevo; estimular la "destrucción creativa" al volver obsoletos los recursos existentes y necesaria su renovación. Tiene un efecto positivo en los aspectos económicos, sociales y ecológicos (Waichung, 2014); (Belz y Binder, 2015). Ideas innovadoras que causan impacto económico y social a través del emprendimiento, posibilitando el crecimiento progresivo no solo en el empleo sino a nivel productivo, económico y social en las comunidades (Duarte y Ruiz, 2009).
La Agencia de los Estados Unidos para el Desarrollo Internacional (USAID) y el Fondo Monetario Internacional (FMI) han promovido iniciativas dirigidas a entender y fomentar el emprendimiento como una manera de enfrentar los negocios en los países en vías de desarrollo (Easterly, 2005). Entretanto la Agenda 2030 para el desarrollo sostenible (Naciones Unidas, 2018), hoja de ruta que promueve las Naciones Unidas como una oportunidad para los países de América Latina y el Caribe para el salto que imponen las actuales circunstancias ha expresado en sus objetivo de desarrollo sostenible (ODS 1 y 12) la necesidad de "Asegurar la sostenibilidad de los sistemas de producción de alimentos y aplicar prácticas agrícolas resilientes que aumenten la productividad y la producción, contribuyan al mantenimiento de los ecosistemas" (p.21), así como de "... lograr un turismo sostenible que cree puestos de trabajo y promueva la cultura y los productos locales." (p.57).

De esta manera se impone la necesidad de realizar estudios en países en desarrollo que posibiliten identificar sus potencialidades para enfrentar estos nuevos desafíos a partir de los recursos endógenos que disponen para su racional y certero aprovechamiento. Perú constituye uno de estos países de la región de América Latina necesitado de catapultar su desarrollo. A tono con los nuevos paradigmas, 
ha apostado al emprendimiento de negocios ecoamigables tomando como punto de partida sus potencialidades. Fue necesario así realizar un estudio diagnóstico, particularmente en Tayacaja, provincia caracterizada por una gran biodiversidad pero también por muy bajos niveles de desarrollo económico y social.

Este trabajo muestra los métodos, resultados y valoraciones en torno a las potencialidades identificadas para el desarrollo de emprendimientos ecoamigables en la provincia de Tayacaja, propuesto como objetivo para este trabajo.

\section{MATERIALES Y MÉTODOS}

Para realizar el diagnóstico, se definió como población los distritos de la provincia de Tayacaja. Para la selección de la muestra se fomentó la participación de líderes de la municipalidad. También se buscó la participación de la mujer como agente en la vida social y productiva de las comunidades en zonas rurales. Participaron en el estudio un total de 128 personas de la comunidad y ocho autoridades o funcionarios de la municipalidad.

Se emplearon como métodos y técnicas de investigación la revisión documental, observación científica, entrevista y encuesta.

Se revisaron documentos oficiales emitidos por el Instituto Nacional de Estadística e Informática (INEI, 2020) así como investigaciones precedentes, para la identificación de las principales características económicas, sociales y ambientales, de la provincia de Tayacaja.

La observación científica fue posible con trabajo de campo en visitas a los diecinueves distritos de la provincia de Tayacaja, con el propósito de constatar las condiciones de vida, así como la situación económica y cultural de sus pobladores. También apreciar características ecosistémicas de la zona. Se tomaron evidencias gráficas de elementos urbanísticos y de actividades económicas de los distritos.
Se realizaron entrevistas (anexo 1) a autoridades y líderes de instituciones sociales con el fin de identificar las problemáticas y potencialidades distritales de la provincia de Tayacaja, así como las aspiraciones de crecimiento económico, social y cultural.

Además, se aplicaron encuestas a miembros de la comunidad presentes en los momentos de las visitas a los distritos. El diseño de esta encuesta consideró varios de los elementos evaluados en la entrevista, de modo que posibilitó complementar los resultados a partir de los diferentes públicos a los que fueron dirigidas estas técnicas.

A partir de los resultados derivados de la aplicación de estas técnicas se calculó el por ciento de participación de los distritos de la provincia; se identificaron las actividades económicas que en ella se desarrollan; capacitaciones recibidas y las demandadas; los emprendimientos actuales y futuros. También se determinaron las fortalezas, oportunidades, debilidades y amenazas (FODA) a partir de los resultados derivados de las técnicas aplicadas, lo que posibilitó diseñar una matriz FODA con su respectiva interpretación.

El procesamiento de los datos recogidos durante las entrevistas y encuestas realizadas para el diagnóstico, se realizó aplicando el software SPSS y el Microsoft Excel para la obtención de los gráficos.

\section{RESULTADOS}

\section{Breve caracterización de la Provincia Taya- caja}

La Provincia de Tayacaja está ubicada en el norte de la región de Huancavelica de Perú. Abarca una superficie de 3,564.50 Km2, que equivale al $16.1 \%$ de la superficie total de la Región. Cuenta con un total de 21 distritos, cinco de ellos de nueva creación (Municipalidad Provincial de Tayacaja, 2015).

Cuenta con una población de 81403 habitantes, En el mapa número 4 se observa la clasificación 
de los distritos de la provincia de Tayacaja según el porcentaje de población analfabeta. Se evidencian distritos con valores de analfabetismo superiores al $25 \%$. En general, más de la mitad de los distritos del territorio provincial presenta valores mayores al $20 \%$, contra un promedio nacional del $5.9 \%$ (INEI, 2018).

De acuerdo con la Meso ZEE de Huancavelica, la provincia de Tayacaja tiene diversos sitios arqueológicos y paisajes culturales que pueden ser considerados parte de los servicios culturales porque son espacios de recreación y educación para la población. Aunque el estado de conservación de muchos de estos sitios, como las pinturas rupestres de Tongos o tramos del Qhápac Ñam, no es el adecuado, han sido considerados dentro del Mapa de los servicios Ecosistémicos. Asimismo, existen diferentes pueblos y zonas de andenería que están considerados como parte del paisaje cultural de la provincia (Gobierno Regional de Huancavelica, 2013).

Desde el punto de vista económico Tayacaja es una zona eminentemente de producción agrícola y ganadera, sin embargo, tal como señala en el Estudio de Diagnóstico y Zonificación de la Provincia de Tayacaja (Gobierno Regional de Huancavelica, 2006), aunque existen condiciones para el cultivo permanente de café, melocotón, palta, durazno, naranja, plátano, limón, mango, entre otras frutas, falta tecnologías para su óptimo aprovechamiento. Así mismo las prácticas inadecuadas para la fertilización de suelos; el desconocimiento de sus pobladores en estos temas y las resistencias culturales para incorporar nuevas y mejores prácticas, son algunos de los factores que afectan un apropiado uso de sus recursos.

Según las proyecciones económicas regionales para el periodo 2018-2019 (MACROCONSULT, 2018) esta provincia deberá beneficiarse con proyectos de Estudio de Diagnóstico y Zonificación de la Provincia de Tayacaja (Gobierno Regional de Huancavelica, 2006) que contribui- rá al PBI de la región en la que se encuentra enclavada,

Los principales indicadores provinciales en base a las informaciones provenientes de las estimaciones y proyecciones poblacionales realizadas por el INEl en el periodo 2007-2018 (INEI, 2020) evidencia algunas de las problemáticas de esta provincia. El analfabetismo, la falta de servicios básicos y el acceso a las nuevas tecnologías pesan de manera significativa en el incremento y profundización de las condiciones de pobreza y exclusión de los procesos de desarrollo de su población, no permitiendo, entre otros, la solución de problemas asociados a los efectos del cambio climático que afecta de manera significativa y directa a sus ecosistemas.

La reducción de estas brechas socioeconómicas supone, entre otros aspectos, el aprovechamiento responsable y sostenible de los recursos existentes en la zona, así como el impulso a actividades económicas más prometedoras y ecológicamente amigables.

\section{Resultados del diagnóstico}

- De acuerdo a las técnicas y métodos empleados durante el estudio se arribaron a los resultados que se exponen.

- Se identificó que la agricultura y la ganadería representan las actividades económicas más importantes de la provincia, sin embargo, la producción es baja y las condiciones en las que se producen están más enfocadas a una agricultura subsistencia. Se realizan ferias en las cuales se ofrecen en venta ganado y productos de los distritos aledaños. Los productos lácteos que se procesan comercialmente en la ciudad de Huancayo y Lima también se comercializan en estas ferias.

- De los 128 participantes en el estudio 74 fueron hombres (58 \%) y 54 mujeres (42\%).

- El porciento de participantes por distritos en relación con el total de la provincia arrojó como más destacados: Pampas con 28 $\%$, seguido de Quichuas y Acraquia con 10 
\% cada uno, resultando estos dos últimos significativamente más bajo que el porciento de Pampas, pero muy superior a los 15 restantes distritos. En la tabla 1 anexo 3 se muestran los porcientos de participación por distritos.

- Tanto los encuestados como los entrevistados declararon un total de diecinueve actividades económicas en los distritos de la provincia (pregunta 1 de la entrevista y 5 de la encuesta). Obsérvese el gráfico (figura 1) donde se evidencia que las actividades más reconocidas por los participantes resultaron: comerciante, producción agrícola y la producción agrícola y ganadera, representando estas tres el $79 \%$ del total de actividades. La tabla 2 anexo 4 se muestran estos resultados.

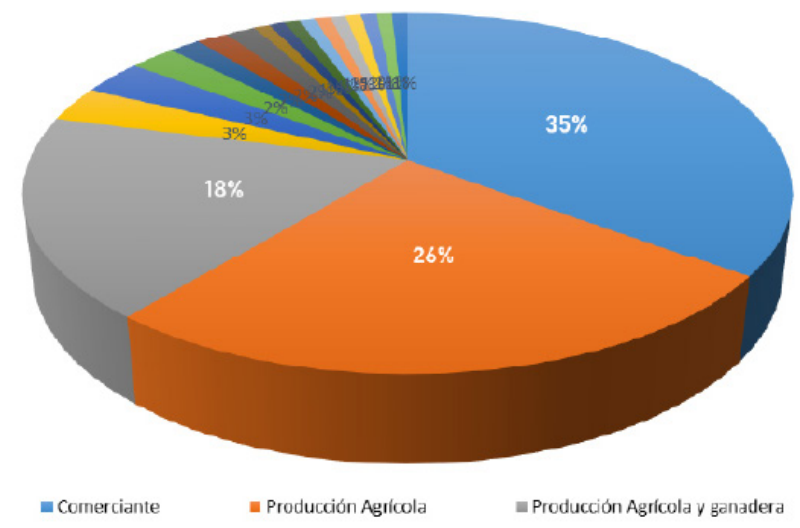

Figura 1. Principales actividades económicas de la provincia de Tayacaja

- A partir de los resultados sobre cuáles son las principales fortalezas, debilidades, oportunidades y amenazas del distrito, indaga- das en las preguntas de la 1 a la 4 de la encuesta y pregunta 2 de la entrevista, se elaboró la siguiente matriz FODA.

Tabla 1. Matriz FODA de la provincia de Tayacaja

\begin{tabular}{ll}
\multicolumn{1}{c}{ FORTALEZAS } & \multicolumn{1}{c}{ OPORTUNIDADES } \\
\hline Diversidad de suelos. & Recursos turísticos con potencial de visita. \\
Agricultura principal actividad & Revaloración de su cultura viva. \\
económica. & \\
Cercanía e interconexión con la ciudad de & Existencia de un marco normativo por el \\
Huancayo. & Ministerio del Turismo en Perú \\
Presencia de la Universidad & Desarrollo artesanal de la provincia. \\
Creación de organizaciones de & Desarrollo de asociaciones ganaderas y \\
emprendimiento & agrícolas \\
Primera región orgánica del Perú & Rol más activo de la mujer en el desarrollo \\
& social. \\
Diversidad de ecosistemas & Impulso a Huancavelica como la primera \\
& región orgánica del Perú \\
& Convocatoria de la (IFOAM 2019) sobre la \\
& comercialización de alimentos libres de \\
& químicos y cultivados de forma amigable.
\end{tabular}




\section{DEBILIDADES}

Negocios incipientes.

Desconocimiento de servicios de calidad.

Desconocimiento de agricultura

sostenible.

Falta de establecimientos de hospedaje.

Carencia de variedad de gastronomía.

Falta de apoyo tecnológico

Carencia de servicios básicos (agua y

desagüe)

\section{AMENAZAS}

Terrorismo.

Cercanía al Vraem.

Migración población joven

Cambio climático y contaminación

ambiental.

Fuente: A partir de los resultados de las técnicas aplicadas durante esta investigación

- Relativo a las asociaciones, su presencia en los distritos y elementos de su funcionamiento indagadas en la entrevista (preguntas 3 y de la 9 a la 12), se identificó que los entrevistados declararon contar con 18 asociaciones en el distrito de Ñahuimpuquio, 45 en el distrito de Acostambo y 84 en el de Daniel Hernández. Estas responden a asociaciones fundamentalmente de producciones agropecuarias. También se pudo conocer que las asociaciones reciben un mayor financiamiento de los proyectos de desarrollo si cuentan con la presencia de mujeres y jóvenes. Asimismo, se identificó que estas asociaciones están vinculadas a proyectos de desarrollo local financiados esencialmente por los gobiernos de las localidades.
- El estudio del emprendimiento de negocios ecoamigables se efectuó analizando lo que existía en el momento del estudio y en torno a cuáles serían las principales propuestas (pregunta 4 de la entrevista).

El análisis por distrito posibilitó identificar que el distrito de Pampas contaba en el momento del estudio con la mayor cantidad de emprendimientos de la provincia (nueve), seguido de Acraquia con cinco, mientras Ahuaycha y Pasos con cuatro cada uno. En estos cuatro distritos se concentraba el $49 \%$ de los emprendimientos que se desarrollaban en la provincia. La Tabla 4 (anexo 5) muestra el comportamiento por cada distrito.

Las propuestas de emprendimientos por distrito aparecen en la Figura 2.

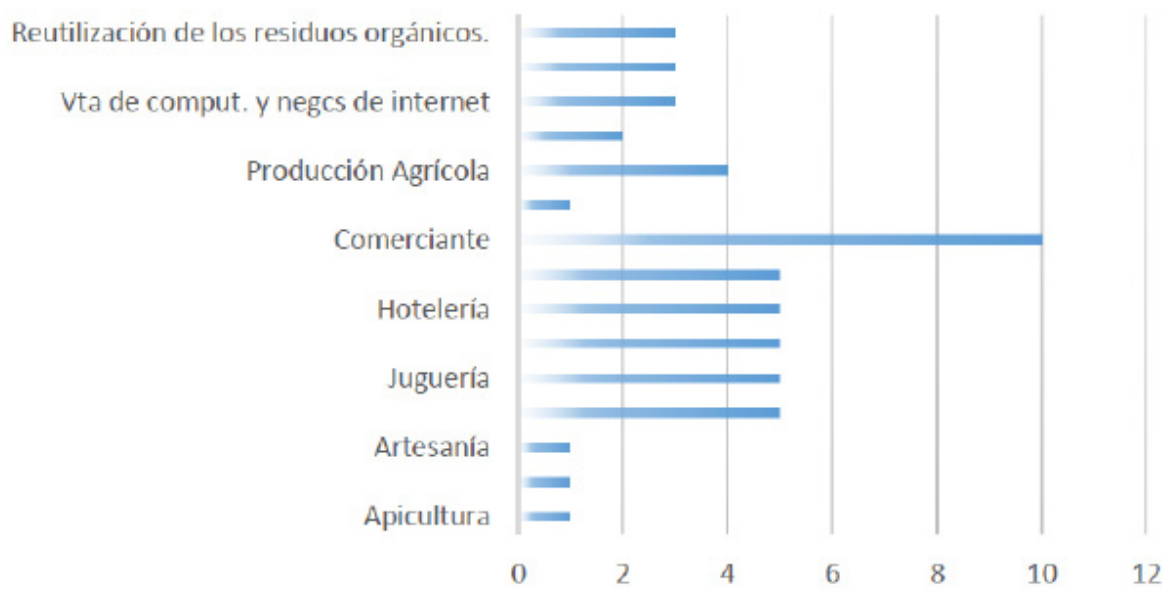

Figura 2. Emprendimientos propuestos en los distritos de la provincia de Tayacaja. 
Nótese que el negocio de Comerciante es la propuesta de emprendimiento por la que más se aboga, 10 de los distritos así lo declararon. Este tipo de negocio se propuso para varios productos agrícolas de la zona (paltas, maca, papas, rocoto y orquídeas), sin embargo se propusieron más la comercialización de productos lácteos y la de huevos. Juguería, restaurantes, hotelería, catering y turismo constituyeron los emprendimientos más citadas después de la comercialización, concentrándose en ellos el 64 \% del total de propuestas.

Un análisis comparativo por distritos reveló el avance o no de los distritos en relación a los emprendimientos que desarrollaban y los que se propusieron. Obsérvese en la figura 3 como Pampas se mantuvo a la vanguardia por la cantidad de nuevos negocios que se propuso emprender (11). También mostraron resultados muy significativos Pichos y Salcabamba con ocho propuestas cada uno, seguidos de Quichuas y Santiago de Tucuma con seis. En todos estos distritos se evidenció un crecimiento significativo en la cantidad de negocios que se propusieron emprender. En cambio, distritos como Acraquia y Pasos mostraron un retroceso al proponerse muchos menos negocios que los ya emprendidos y cinco de los distritos no se propusieron emprender nuevos negocios ni crecer en los existentes.

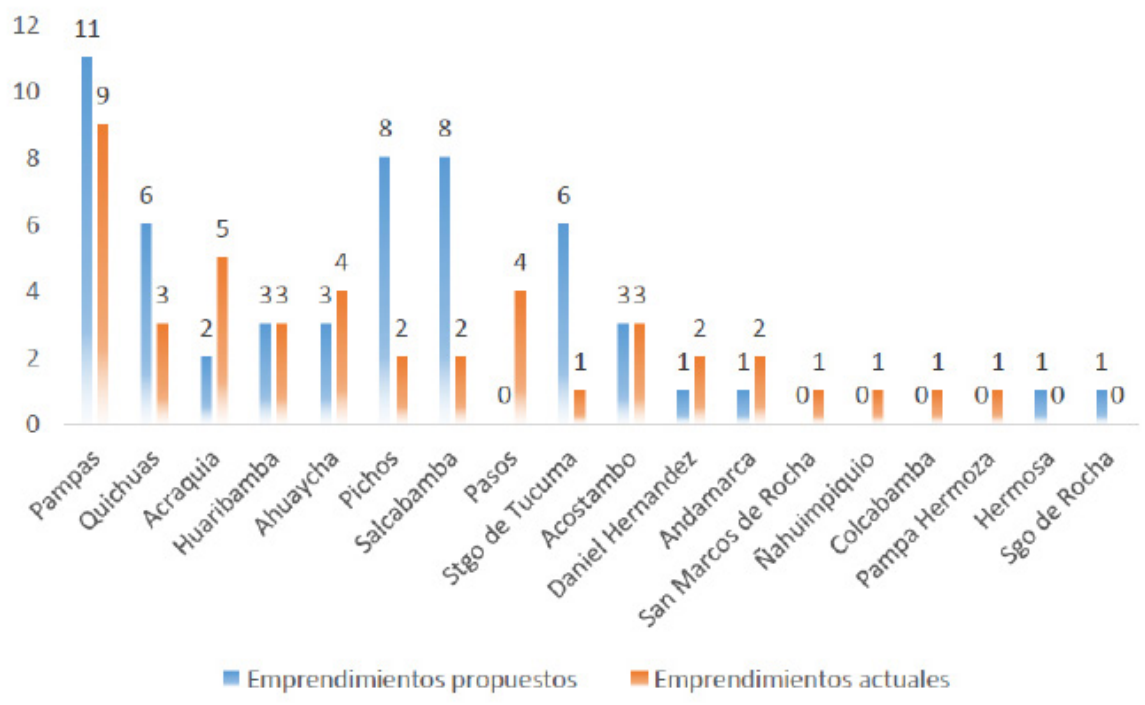

Figura 3. Emprendimientos propuestos en los distritos de la provincia de Tayacaja.

También se identificaron qué tipos de nuevos negocios proponían emprender los distritos y en cuáles de los existentes crecer. Resultaron significativos: Pampas con la propuesta de ocho nuevos tipos de negocios y crecer en tres de los ya existentes (Hotelería, Turismo y Comercialización); Pichos se propuso ocho nuevos negocios y Salcabamba siete nuevos y el crecimiento en el Turismo; Quichuas cinco nuevos y crecer en la Hotelería; mientras Santiago de Tucuma se propuso seis nuevos negocios.
- Los principales problemas (pregunta 6 de la encuesta), se concentraron en falta de capital (43\%), falta de capacitación (24 \%) y falta de tecnología (20\%). El $13 \%$ restante agrupó gran variedad de problemas, por lo que no se consideró representativo para el estudio.

- Las preguntas 7 y 8 de la encuesta y 7 de la entrevista buscaban información relativa a la cantidad de personas capacitadas y los temas recibidos. Se pudo determinar 
que de los 128 participantes solo el 48 \% había recibido capacitación; los temas recibidos estuvieron relacionados esencialmente con la producción agrí- cola (66\% del total de temas recibidos); mientras los relacionados con la actividad ganadera representaron el $15 \%$ (ver figura 4).

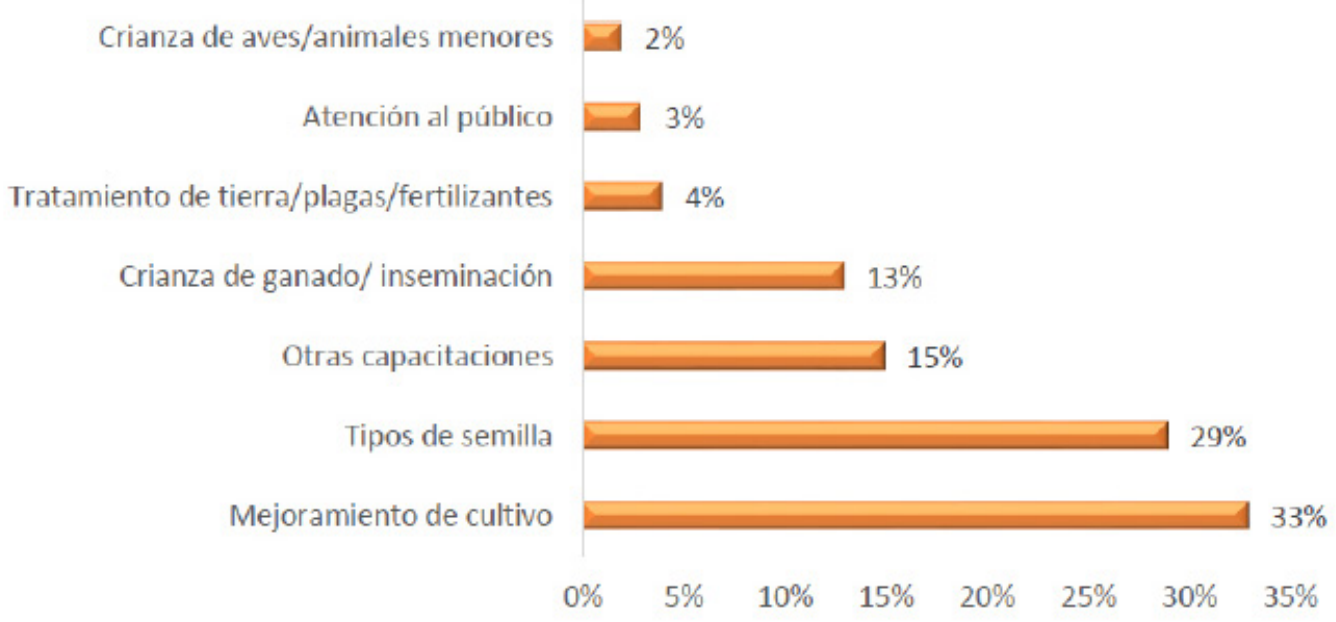

Figura 4. Principales temas de capacitación recibidos por los participantes Los temas recibidos guardan correspondencia con las principales actividades económicas que caracterizan la zona.

- En relación con los temas en los que consideraban debían capacitarse (preguntas 8 de la entrevista y 9 de la encuesta), los participantes declararon la gestión, mejora y creación de nuevos negocios con más del $80 \%$. También se demandaron temas relacionados con la comercialización, marketing y publicidad. En la figura 5 se exhiben estos resultados,

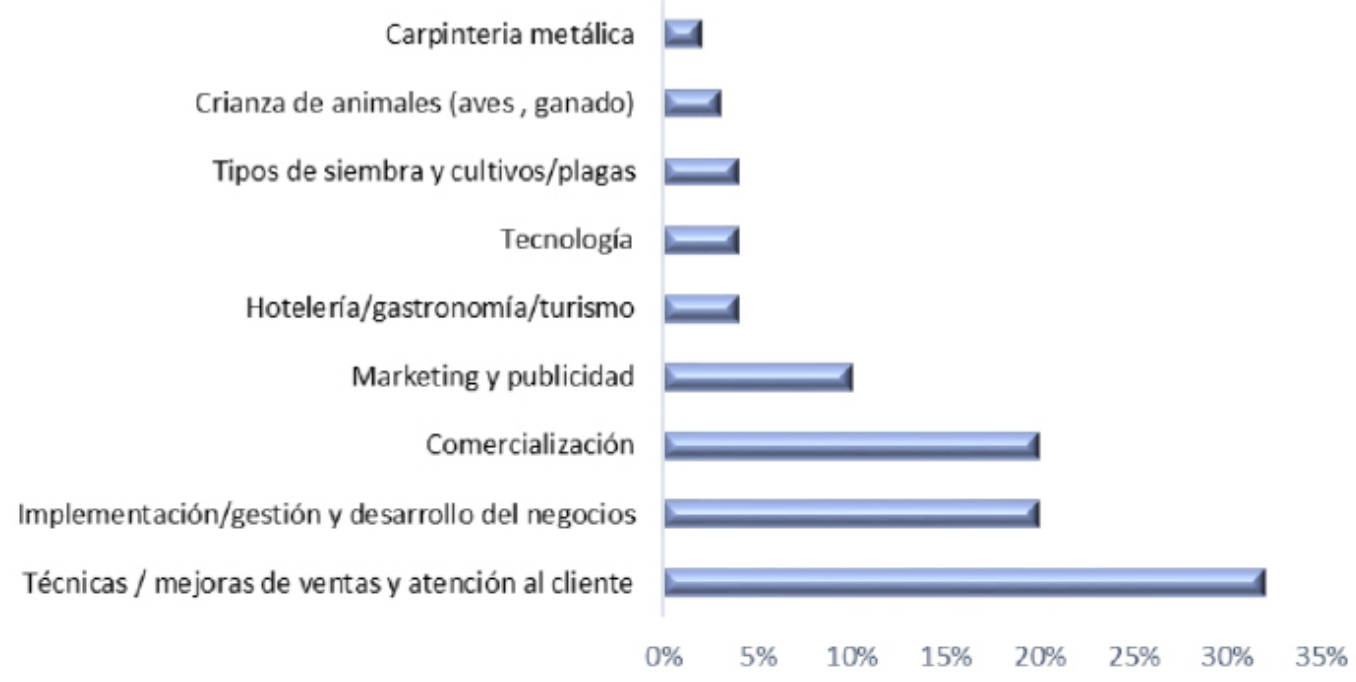

Figura 5. Principales temas de capacitación demandados por los participantes 
Los temas de capacitación más demandados guardan cierta correspondencia con los nuevos negocios que se propusieron emprender. La atención al cliente, temas relacionados con la implementación, gestión y desarrollo de nuevos negocios, comercialización, marketing y publicidad responden a las necesidades de conocimientos y capacidades humanas que se requieren para emprender los negocios propuestos.

\section{DISCUSIÓN}

(Borea, 2006), (Ulate, 2010) y (Buckland y Murillo, 2014), sostienen que la innovación genera la creación de empresas, lo que junto con el emprendimiento, promueven la creación de empleo, competitividad, oportunidades y crecimiento económico en las regiones. (Curbelo y Peña, 2012; Duarte y Ruiz, 2009). En particular las actividades emprendedoras productivas contribuyen de una manera constante al desarrollo de nuevos mercados y a su posterior evolución, del mismo modo que a la evolución de los mercados ya existentes. (Gancia y Zilibotti, 2005), (Minniti y Lévesque, 2008).

Innovar no es suficiente para considerar un negocio como un emprendimiento. La creatividad para aprovechar oportunidades, generar riquezas en condiciones de incertidumbre, asumir riesgos, el optimismo y la perseverancia deben caracterizar estos procesos. (Amaru, 2008).

Partiendo de estas afirmaciones y los resultados obtenidos en el diagnóstico realizado en este trabajo se reflexionó en torno a las posibilidades de fomentar emprendimientos de negocios ecoamigables en la provincia Tayacaja.

El hecho que el $79 \%$ de las actividades económicas se concentren en la producción ganadera y agrícola, así como en la comercialización, se corresponde con las características biodiversas de los ecosistemas y las identi- dades culturales de la zona. También por el hecho de que estas actividades hayan sido favorecidas por proyectos de desarrollo local, de allí que las principales tecnologías y capacitaciones recibidas estuvieran orientadas a potenciar su desarrollo. Sin embargo, se pudo apreciar que las actividades agropecuarias se dirigen esencialmente al uso doméstico, lo que evidencia escasa visión de sus pobladores en cuanto a las potencialidades de éstas para el encadenamiento con otras actividades económicas. Estas fortalezas naturales, económico y culturales, articuladas de manera innovadora, podrían constituir oportunidades de emprendimiento de nuevos negocios, lo que supondría mejora de la calidad de vida, generación de fuentes de empleo y una contribución al desarrollo económico de la comunidad. Además constituiría una vía para mitigar el éxodo de jóvenes, problemática de migración generada, entre otros, por la falta de oportunidades laborales, según afirma (Mata, 2014).

De acuerdo a las raíces culturales de los comunitarios, la artesanía podría convertirse también en otra oportunidad de emprendimiento. La incorporación de nuevas técnicas para su producción y comercialización a través de industrias locales como generadoras de empleo y recursos económicos (Oluwayemisi y Fillis, 2016), y su encadenamiento innovador con otras actividades como el turismo, soportado con agencias de viajes, alojamientos en casas particulares, servicios de restaurantes podrían generar nuevos y atractivos emprendimientos.

Según estudios de (Barragán y Ayaviri, 2017) la asociatividad y la economía solidaria, unido a la innovación y el emprendimiento, se encuentran entre los elementos más importantes que contribuyen al desarrollo local. Sin la organización efectiva de relaciones de colaboración, los eslabones de la cadena de valor se debilitan, y como resultado todos los componentes son menos eficaces en sus funciones individuales, lo que hace que la coopera- 
ción sea uno de los factores más importantes (Tapia et. al, 2015).

En tal sentido la provincia concentra sus asociaciones en los distritos Ñahuimpuquio, Acostambo, Daniel Hernández y Colcabamba, los que podrían resultar privilegiados en relación con los restantes distritos por los aportes financieros que reciben estas asociaciones de los proyectos de desarrollo local, financiamiento externo que fue identificado por (García, 2013) como uno de los elementos determinantes para los procesos de innovación. Estos distritos también podrían resultar beneficiados con nuevas tecnologías y capacitaciones a sus asociados, lo que su vez se traducirá en desarrollo de capacidades para la producción de alimentos sanos, la protección del ecosistema de la zona y el emprendimiento de nuevos negocios.

Por otra parte, la presencia de asociaciones se convierte a su vez en un incentivo para la creación de nuevas asociaciones o el fortalecimiento de las ya existentes. Esta proliferación de asociaciones presupone más financiamientos, los que se incrementan cuando se garantiza la participación de mujeres y jóvenes. Implica entonces, no sólo mayores beneficios económicos y capacitación, también mayor inclusión social e igualdad de género, inserción de nuevas tecnologías, más soluciones innovadoras y la generación de nuevas capacidades en los habitantes para el emprendimiento de nuevos negocios.

De acuerdo a lo expresado por (Duran, 2016), a partir de la articulación de varias asociaciones, se generaría otra superior de tipo organizativo con el propósito de agregar intereses, y de esta manera alcanzar objetivos mayores, tanto para los miembros de las asociaciones como para el sector productivo al que pertenecen. De lo anterior se desprende la necesidad de incrementar la cantidad de asociaciones en la provincia, así como de la articulación entre ellas como vías para lograr mayores beneficios económicos, sociales y ambientales tanto para los pobladores como para la provincia.

A través del cuidado del medio ambiente, el potenciamiento de los emprendimientos de turismo comunitario, comercio justo local e internacional, pasantías y transferencia de tecnología de campesino a campesino, la organización se abre al mundo y al comercio solidario integrando a todas sus organizaciones locales en este proceso para mejorar la calidad de vida de sus habitantes a través de los emprendimientos (Mata, 2014). Se deriva entonces que la diversidad de suelos y de ecosistemas que posee Tayacaja y el empleo de tecnologías limpias y amigables con el entorno, además de una contribución al cuidado del medio ambiente, se convierten en un potencial para la producción de alimentos sanos y sostenibilidad en su suministro para ofertas gastronómicas variadas en un entorno ecoamigable, convirtiéndolo así en un atractivo turístico y una oportunidad de empredimiento. Todo ello implicará garantía de los servicios hidráulicos, la aplicación de nuevas tecnologías, la creación de nuevas y variadas capacidades de hospedaje y el impulso a los negocios incipientes.

(Galindo-Martín, Méndez-Picazo, y Castaño-Martínez, 2016) apuntan que los emprendedores, gracias al empleo de sus propios recursos o de los que obtienen en el mercado y a las innovaciones que generan o introducen, posibilitan, junto a otros agentes sociales, la creación de la riqueza que luego se repartirá entre los distintos componentes de la sociedad. En este sentido una serie de acciones concertadas y coordinadas con las políticas públicas regionales y provinciales orientadas a la creación y puesta en marcha de servicios ecosistémicos resulta ser prioritaria para apoyar, atender, capacitar y acompañar la población en enfrentar los desafíos, redireccionando para ello los ejes de desarrollo que conduzcan a la reducción de las brechas socioeconómicas que sufren. 
Contar con una Universidad en la provincia puede constituir una palanca de desarrollo local que trasciende la dimensión humana, a través de su actividad formadora, para convertirse en desarrolladora de capacidades en los individuos, organizaciones y comunidades. Su participación activa y armoniosamente articulada en la aplicación de la ciencia, la tecnología y la innovación, junto con otros agentes como las empresas y el gobierno de la provincia, reconocida por Castillo 2010, como la triple Hélice, pueden catapultar al desarrollo económico y social de la provincia a partir del aprovechamiento de las potencialidades de la zona donde se encuentra enclavada.

Para el desarrollo del turismo, se puede considerar algunas propuestas que podrían ser ejecutadas por el sector público y privado según el Informe Final del Inventario turístico y propuesta de desarrollo científico y comercial del turismo en el distrito de Pampas.

- Establecer una política para el desarrollo turístico en la provincia de Tayacaja.

- Realizar coordinación interinstitucional entre el gobierno local y el MINCETUR.

- Enfocar el PENTUR, para que de manera específica el gobierno local logre elaborar el PERTUR (Plan Estratégico Regional de Turismo). Siempre con asistencia del MINCETUR o profesionales del sector turismo.

- Ejercer rol ejecutor de las inversiones y acciones necesarias para el desarrollo definidas en un plan estratégico y operativo.

\section{CONCLUSIONES}

1. La biodiversidad de los ecosistemas, las identidades culturales y las actividades económicas que caracterizan la provincia de Tayacaja constituyen importantes potencialidades para su desarrollo, sin embargo, los escasos financiamientos externos, la resistencia a la introducción de nuevas tecnologías y buenas prácticas por parte de sus comunitarios, así como los vacíos de capacitación de éstos en temas asociados al lanzamiento de nuevos negocios limitan la percepción de oportunidades de emprendedurismos ecoamigables en la Provincia.

2. Las asociaciones de productores y comerciantes se encuentran entre los elementos más importantes que contribuyen al desarrollo local pues garantizan la entrada de financiamiento externo, promueven la inclusión social e igualdad de género, aplicación de nuevas tecnologías y soluciones innovadoras, la generación de nuevas capacidades en los habitantes para el emprendimiento de nuevos negocios, de allí la necesidad de fomentar su expansión en Tayacaja, dado su limitada presencia en los distritos de la provincia.

3. La Universidad como formadora de capacidades y su quehacer apropiadamente articulado con las empresas y el gobierno para la introducción de la ciencia y la técnica, la convierten en un potencial agente dinamizador en el desarrollo socioeconómico de la provincia y un actor esencial para el emprendimiento de nuevos negocios.

\section{AGRADECIMIENTOS}

Los investigadores desean expresar su agradecimiento a la Universidad Nacional Autónoma de Tayacaja "Daniel Hernández Morillo" de la Región Huancavelica y al Fondo de Desarrollo Socioeconómico de Camisea (FOCAM) por financiar la investigación en el marco del proyecto "Desarrollo de un sistema para la aplicación de modelos de análisis territorial y adaptación al cambio climático con tecnología Smart Environment" aprobado con Resolución de Comisión Organizadora N 0122-2018-CO-UNAT.

\section{REFERENCIAS BIBLIOGRÁFICAS}

Amaru, A. C. (2008). Administración para emprendedores. Fundamentos para la creación y 
gestión de nuevos negocios. Estado de México, México: Pearson Educación de México, S.A. de C.V.

Barragán, M. C., y Ayaviri, V. D. (2017). Innovación y Emprendimiento, y su relación con el Desarrollo Local del Pueblo de Salinas de Guaranda, Provincia Bolívar, Ecuador. Información Tecnológica, 28(6), 71-80 doi:http://dx. doi.org/10.4067/S0718-07642017000600009

Belz, F., y Binder, J. (2015). Sustainable Entrepreneurship: A Convergent Process Model. Wiley Online Library, 1, 1- 17.

Borea, F. (2006). Innovación y Desarrollo Económico. Hologramática, 2(4), 1-19

Buckland, H., y Murillo, D. (2014). La Innovación Social en América Latina. Instituto de Innovación Social, 75, 1-71.

Castillo, H. G. C. (2010). El modelo de la triple hélice como un medio para la vinculación entre la universidad y empresa. Revista Nacional de administración, 1(1), 85-94.

Curbelo, J. L., y Peña, I. (2012). Emprendimiento y Competitividad Regional. Boletín de Estudios Económicos, 67(205), 59-76.

Duarte, T., y Ruiz, M. (2009). Emprendimiento, Una Opción para el Desarrollo. Scientia et Technica, 3(43), 326-331.

Easterly, W. (2005). What did structural adjustment adjust? The association of policies and growth with repeated IMF and World Bank adjustment loans. Journal of Development Economics, 76(no 1), pp. 1.

Galindo-Martín, M. A., Méndez-Picazo, M. T., y Castaño-Martínez, M. (2016). Crecimiento, Progreso Económico y Emprendimiento, (1) 62-68 (2016). Journal of Innovation \& Knowledge, 1(1), 62-68. doi:http://dx.doi.org/10.1016/j.jik.2016.01.006,
Gancia, G., y Zilibotti, F. (2005). Horizontal innovation in the theory of growth and development. . Handbook of Economic Growth, 1 A(Elsevier).

García, D. (2013). Financiación de la innovación en las Mypyme Latinoamericanas. Estudios Gerenciales, 29(2013), 12-16.

Gobierno Regional de Huancavelica. (2013) Meso Zonificación Ecológica y Económica del Departamento de Huancavelica.

Gobierno Regional de Huancavelica. (2006). Estudio de Diagnóstico y Zonificación para el tratamiento de la Demarcación Territorial de la Provincia de Tayacaja.

INEI. (2018). Resultados definitivos de lo Censos Nacionales 2017 - Huancavelica. Lima.

INEI. (2020). PERU Instituto Nacional de Estadística e Informática INEI. Retrieved from http://www.inei.gob.pe/

MACROCONSULT. (2018). Reporte Económico "Proyecciones económicas por región 20182019". Retrieved from https://sim.macroconsult.pe/proyeccionesregionales/

Mata, O. (2014). Los Proyectos Solidarios de Salinas de Guaranda y su Aporte para la Construcción de "Otra Economía". (Biblioteca Digital de Vanguardia, para la Investigación en Ciencias Sociales, Región Andina y América Latina, Tesis de Maestría ), Universidad Nacional de Chimborazo, Ecuador. Retrieved from goo.gl/ LYq3XT

Minniti, M., y Lévesque, M. (2008). Recent Developments in the Economics of Entrepreneurship. . Journal of Business Venturing, 23(6), pp. 603-612.

Municipalidad Provincial de Tayacaja. (2015). Plan de Desarrollo Concertado Tayacaja 2015 - 2021. Pampas. 
Naciones Unidas. (2018). La agenda 2013 y los objetivos de desarrollo sostenible. Una oportunidad para América Latina y el Caribe Santiago: Santiago.

Oluwayemisi, A., y Fillis, I. (2016). The Role of Handicraft micro Enterprises as a Catalyst for Youth Employment. Business School, 2, 1-17. doi:http:// dx.doi.org/10.1080/17510694.2016.1247628.

Schumpeter, J. (1942). Capitalismo, socialismo y democracia.

Ulate, I. (2010). La Innovación que potencia el desarrollo. Revista Nacional de Administración, 1(2), 79-86.

Waichung, H. (2014). Regional Development in the Global Economy: A Dynamic Perspective of Strategic Coupling in Global Production Networks. Regional Science Policy, 1, 1-24

\section{CORRESPONDENCIA:}

MsC. Ronald Ortecho Llanos

ronaldortecho@unat.edu.pe 